\title{
Deeply Virtual Compton Scattering at HERA and Prospects on Nucleon Tomography
}

\author{
Laurent Schoeffel \\ CEA Saclay, DAPNIA-SPP, 91191 Gif-sur-Yvette Cedex, France
}

\begin{abstract}
Standard parton distribution functions contain neither information on the correlations between partons nor on their transverse motion, then a vital knowledge about the three dimensional structure of the nucleon is lost. Hard exclusive processes, in particular DVCS, are essential reactions to go beyond this standard picture. In the following, we examine the most recent data and their implication on the quarks/gluons imaging (tomography) of the nucleon.
\end{abstract}

\section{Introduction}

Measurements of the deep-inelastic scattering (DIS) of leptons and nucleons, $e+p \rightarrow e+X$, allow the extraction of Parton Distribution Functions (PDFs) which describe the longitudinal momentum carried by the quarks, anti-quarks and gluons that make up the fast-moving nucleons. These functions have been measured over a wide kinematic range in the Bjorken scaling variable $x_{B j}$ and the photon virtuality $Q^{2}$. While PDFs provide crucial input to perturbative Quantum Chromodynamic (QCD) calculations of processes involving hadrons, they do not provide a complete picture of the partonic structure of nucleons. In particular, PDFs contain neither information on the correlations between partons nor on their transverse motion, then a vital knowledge about the three dimensional structure of the nucleon is lost. Hard exclusive processes, in which the nucleon remains intact, have emerged in recent years as prime candidates to complement this essentially one dimentional picture [2].

The simplest exclusive process is the deeply virtual Compton scattering (DVCS) or exclusive production of real photon, $e+p \rightarrow e+\gamma+p$. This process is of particular interest as it has both a clear experimental signature and is calculable in perturbative QCD. The DVCS reaction can be regarded as the elastic scattering of the virtual photon off the proton via a colourless exchange, producing a real photon in the final state 2, 3, 4. In the Bjorken scaling regime, QCD calculations assume that the exchange involves two partons, having different longitudinal and transverse momenta, in a colourless configuration. These unequal momenta or skewing are a consequence of the mass difference between the incoming virtual photon and the outgoing real photon. This skewedness effect can be interpreted in the context of generalised parton distributions (GPDs) [5, 6, 7]. These functions carry information on both the longitudinal and the transverse distribution of partons. The DVCS cross section depends, therefore, on GPDs [5, 6, 7.

In the following, we examine the most recent data recorded from the DESY ep collider at HERA and their implication on the quarks/gluons imaging of the nucleon [3, 4].

\section{Latest News from the Front}

The first measurements of DVCS cross section have been realised at HERA within the H1 and ZEUS experiments [3, 4. These results are given in the specific kinematic domain of both experiments, at low $x_{B j}\left(x_{B j}<0.01\right)$ but they take advantage of the large range in 


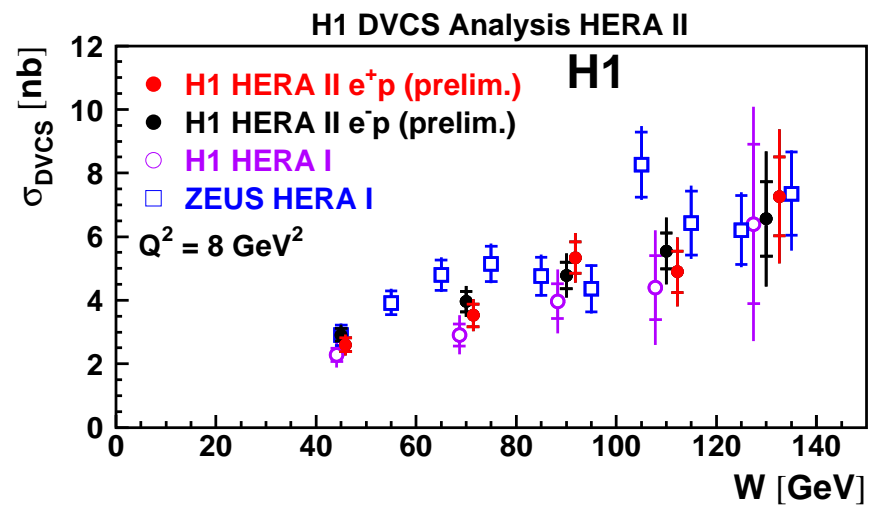

Figure 1: DVCS cross section for positrons/electrons samples as a function of $W$.

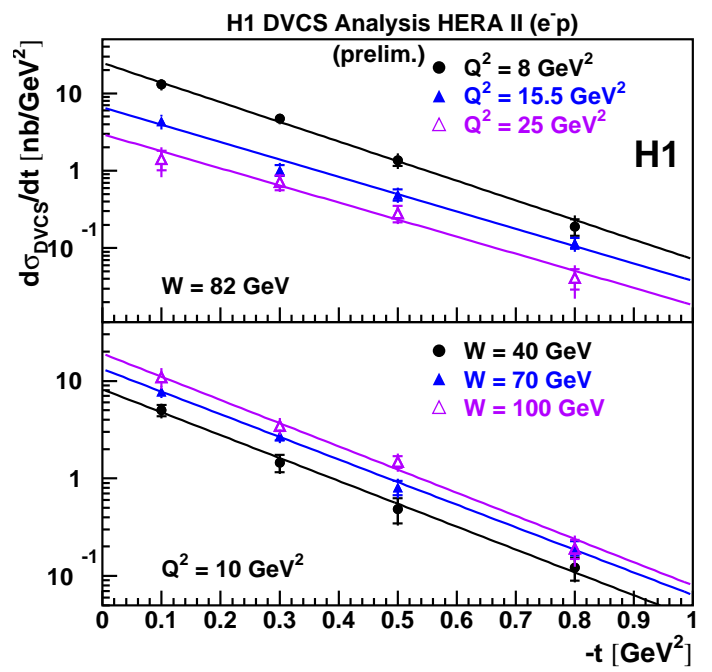

Figure 2: DVCS cross section, differential in $t$ presented with a fit of the form $e^{-b|t|}$.

$Q^{2}$, offered by the HERA kinematics, which covers more than 2 orders of magnitude, from 1 to $100 \mathrm{GeV}^{2}$. It makes possible to study the transition from the low $Q^{2}$ non-perturbative region (around $1 \mathrm{GeV}^{2}$ ) towards higher values of $Q^{2}$ where the higher twists effects are lowered (above $10 \mathrm{GeV}^{2}$ ). The last DVCS cross sections as a functon of $W \simeq \sqrt{Q^{2} / x}$ are presented on figure 1.

A major experimental achievement of $\mathrm{H} 1$ [3] has been the measurement of DVCS cross sections, differential in $t=\left(p^{\prime}-p\right)^{2}$, the momentum transfer (squared) at the proton vertex. Some results are presente on figure 2, we observe the good description of $d \sigma_{D V C S} / d t$ by a fit of the form $e^{-b|t|}$. Hence, an extraction of the $t$-slope parameter $b$ is accessible for different values of $Q^{2}$ and $W$ (see figure 3). 

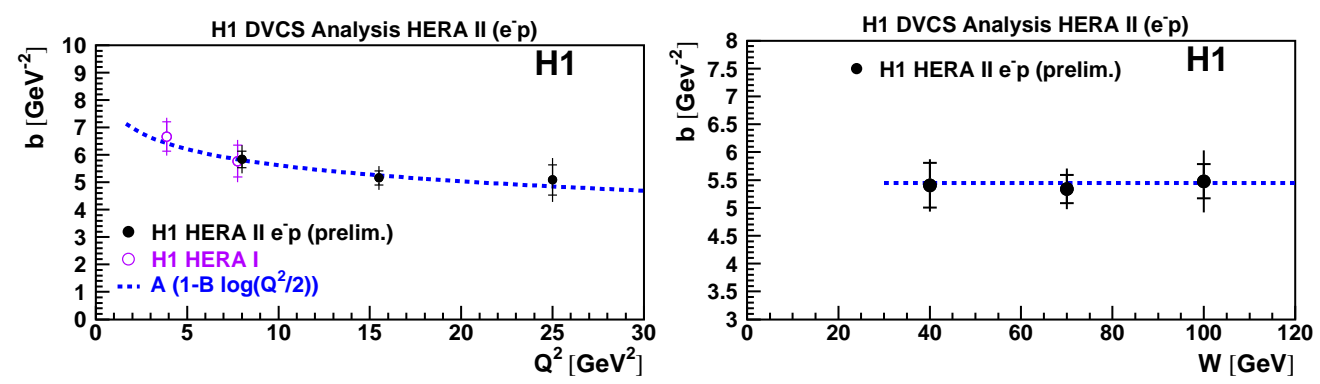

Figure 3: The logarithmic slope of the $t$ dependence for DVCS exclusive production, $b$ as a function of $Q^{2}$ and $W$, extracted from a fit $d \sigma / d t \propto \exp (-b|t|)$ where $t=\left(p-p^{\prime}\right)^{2}$.

\section{Nucleon Tomography}

Measurements of the $t$-slope parameters $b$ are key measurements for almost all exclusive processes, in particular DVCS. Indeed, a Fourier transform from momentum to impact parameter space readily shows that the $t$-slope $b$ is related to the typical transverse distance between the colliding objects [7. At high scale, the $q \bar{q}$ dipole is almost point-like, and the $t$ dependence of the cross section is given by the transverse extension of the gluons (or sea quarks) in the proton for a given $x_{B j}$ range. More precisely, from the generalised parton distribution $G P D$ defined in the introduction, we can compute a parton density which also depends on a spatial degree of freedom, the transverse size (or impact parameter), labeled $R_{\perp}$, in the proton. Both functions are related by a Fourier transform

$$
P D F\left(x, R_{\perp} ; Q^{2}\right) \equiv \int \frac{d^{2} \Delta_{\perp}}{(2 \pi)^{2}} e^{i\left(\Delta_{\perp} R_{\perp}\right)} G P D\left(x, t=-\Delta_{\perp}^{2} ; Q^{2}\right) .
$$

Thus, the transverse extension $\left\langle r_{T}^{2}\right\rangle$ of gluons (or sea quarks) in the proton can be written as

$$
\left\langle r_{T}^{2}\right\rangle \equiv \frac{\int d^{2} R_{\perp} P D F\left(x, R_{\perp}\right) R_{\perp}^{2}}{\int d^{2} R_{\perp} P D F\left(x, R_{\perp}\right)}=4 \frac{\partial}{\partial t}\left[\frac{G P D(x, t)}{G P D(x, 0)}\right]_{t=0}=2 b
$$

where $b$ is the exponential $t$-slope. Measurements of $b$ presented in figure 3 corresponds to $\sqrt{r_{T}^{2}}=0.65 \pm 0.02 \mathrm{fm}$ at large scale $Q^{2}$ for $x_{B j}<10^{-2}$. This value is smaller that the size of a single proton, and, in contrast to hadron-hadron scattering, it does not expand as energy $W$ increases. This result is consistent with perturbative QCD calculations in terms of a radiation cloud of gluons and quarks emitted around the incoming virtual photon.

\section{Beam Charge Asymmetry and Conclusions}

The complete parton imaging in the nucleon would need to get measurements of $b$ for several values of $x_{B j}$, from the low $x_{B j}<0.01$ till $x_{B j}>0.1$. Experimentally, it appears to be impossible. Is it the breakout of quark and gluon imaging in the proton? In fact, there is one way to recover $x_{B j}$ and $t$ correlations over the whole $x_{B j}$ domain: we need to measure a Beam Charge Asymmetry (BCA) 2, 5 .

The determination of a cross section asymmetry with respect to the beam charge is realised by measuring the ratio $\left(d \sigma^{+}-d \sigma^{-}\right) /\left(d \sigma^{+}+d \sigma^{-}\right)$as a function of $\phi$ 2, 5. The lattest experimental result from $\mathrm{H} 1$ is presented in figure 4 with a fit in $\cos \phi$. After applying a deconvolution method to account for the resolution on $\phi$, the coefficient of the $\cos \phi$ dependence is found to be $p_{1}=0.17 \pm 0.03$ (stat.) \pm 0.05 (sys.). 
The relative large positve value (more than two standard deviations from zero) is favoring a non-factorised approach between $x_{B j}$ and $t$ variables, which means that on a wide range of $x_{B j}$, the $t$-slope $b$ is certainly dependent on $x_{B j}$.

This result represents obviously a major progress in the understanding of the very recent field of the parton imaging in the proton. We are at the hedge of the giving a new reading on the most fundamental question to know how the proton is built up by quarks and gluons...

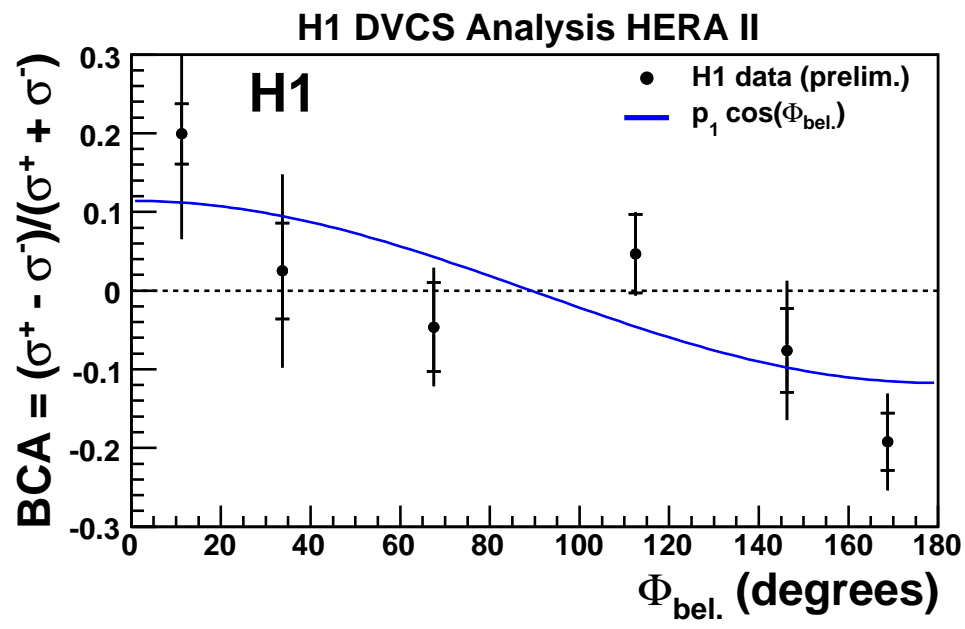

Figure 4: Beam charge asymmetry as a function of $\phi[2]$.

\section{References}

[1] Slides: http://indico. cern. ch/getFile.py/access? contribId=296\&sessionId=31\&res Id=0\&materialId=slides\&conf Id=24657

[2] L. Schoeffel, proceedings of 27th International Conference on Physics in Collision (PIC 2007), Annecy, France, 26-29 Jun 2007. arXiv:0707.3706 [hep-ph].

[3] F. D. Aaron et al. [H1 Collaboration], Phys. Lett. B 659 (2008) 796 arXiv:0709.4114 [hep-ex]] ; A. Aktas et al. [H1 Collaboration], Eur. Phys. J. C 44, 1 (2005) hep-ex/0505061] ; C. Adloff et al. [H1 Collaboration], Phys. Lett. B 517 (2001) 47 arXiv:hep-ex/0107005.

[4] S. Chekanov et al. [ZEUS Collaboration], Phys. Lett. B 573, 46 (2003) hep-ex/0305028].

[5] A. Freund, Phys. Rev. D 68 (2003) 096006 hep-ph/0306012.

[6] L. Schoeffel, Phys. Lett. B 658 (2007) 33. arXiv:0706.3488 [hep-ph].

[7] M. Burkardt, Int. J. Mod. Phys. A 18 (2003) 173 hep-ph/0207047]. 